\title{
Sociodemographic risk factors of alcohol drinking in Hong Kong adolescents
}

\author{
Rong Huang, ${ }^{1}$ Sai Yin Ho, ${ }^{1}$ Man Ping Wang, ${ }^{2}$ Wing Sze Lo, ${ }^{1}$ Tai Hing Lam ${ }^{1}$
}

'School of Public Health, The University of Hong Kong, Hong Kong, China ${ }^{2}$ School of Nursing, The University of Hong Kong, Hong Kong, China

Correspondence to Dr Sai Yin Ho, School of Public Health, The University of Hong Kong, 21 Sassoon Road, Pokfulam, Hong Kong; syho@hku.hk

Received 29 July 2015 Revised 11 September 2015 Accepted 2 October 2015
To cite: Huang R, Ho SY, Wang MP, et al. J Epidemiol Community Health Published Online First: [please include Day Month Year] doi:10.1136/jech-2015206418

\begin{abstract}
Background Adolescent drinking in Western countries has been associated with older age and high socioeconomic status, but the association with family structure was inconsistent.

Methods In a 2012-2013 school-based survey in Hong Kong, 23096 students (mean age 14.7 years, SD 1.8 years) completed an anonymous questionnaire. Current drinking was defined as any drinking and binge drinking as consuming at least 5 drinks on one occasion, both in the past 30 days. Multilevel logistic regression was used to examine the association of sociodemographic characteristics with current drinking, binge drinking and type of alcohol consumed.

Results Current drinking was associated with age ( $\geq 15$ vs $\leq 14$ years) (adjusted OR 1.87, 95\% Cl 1.74 to 2.02), higher perceived family affluence versus low affluence $(1.11,1.02$ to 1.21 for medium affluence; $1.55,1.38$ to 1.75 for high affluence), private housing versus public housing $(1.11,1.01$ to 1.21$)$ and nonintact family versus intact family $(1.31,1.19$ to 1.45 for separated/divorced parents; $1.40,1.21$ to 1.62 for one or both deceased parents). Similar risk factors were observed for binge drinking. Girls were more likely to drink fruit wine $(1.48,1.36$ to 1.62$)$, while they were less likely to drink beer $(0.85,0.79$ to 0.92$)$ and spirits $(0.69,0.54$ to 0.87$)$. Students who reported high family affluence were more likely to drink wine $(1.91,1.59$ to 2.30) and spirits (2.23, 1.54 to 3.24$)$.

Conclusions Generally, adolescents who were older and had higher socioeconomic status were more likely to drink. High family affluence was associated with wine and spirits drinking. Beer and spirits were preferred more by boys, and fruit wine by girls. These results indicated high-risk groups for adolescent alcohol interventions.
\end{abstract}

\section{INTRODUCTION}

Underage drinking is an important public health concern due to its adverse health and social effects, such as intoxication, injuries/violence, drunk driving and unprotected sex. ${ }^{1}$ Binge drinking is a common drinking pattern among adolescents, often leading to fighting, taking or being taken advantage of sexually, vandalism and drug use. ${ }^{2-4}$ Long-term heavy drinking would even lead to mental illnesses and irreversible brain damage. ${ }^{5} 6$

Adolescence is an important period when alcohol drinking habits are formed. ${ }^{7-9}$ Identifying high-risk groups of adolescent drinking has implications on public health interventions. However, studies that specifically examine sociodemographic risk factors of adolescent drinking are few and mostly based on Caucasians. Previous studies have found inconsistent sex differences in adolescent drinking, while older age has predicted drinking. ${ }^{4} 10$ Drinking has been associated with parental professional occupation in American adolescents and family affluence in Slovakian adolescents. ${ }^{11}{ }^{12}$ Non-intact family structure was associated with adolescent drinking in two studies from the USA and Hong Kong, ${ }^{13} 14$ but null findings have also been reported. ${ }^{15}$ Sociodemographic correlates of alcohol drinking in Hong Kong adolescents have seldom been reported, but one study among Hong Kong adults has found weekly drinking associated with male sex, primary education or below, and separated or widowed marital status. ${ }^{16}$

While adults commonly drink several types of alcohol such as beer, wine and spirits, adolescents often have a preference for certain types of alcohol. ${ }^{17}$ Beer is popular among young people, partly due to its link with sports and entertainment put forth by the alcohol industry. ${ }^{18}$ A recent study has shown Australian adolescent girls preferred premixed spirits (mixture of spirits with fruit juice or soft drinks), while boys preferred spirits and beer. ${ }^{19}$ Drinking patterns have been linked to specific alcohol types. ${ }^{17}$ Adolescents with a preference for beer or spirits may drink more and were more likely to experience negative consequences. ${ }^{20}$ However, little is known about the sociodemographic correlates of the type of alcohol consumed.

Hong Kong is the most westernised city of China. Although alcohol drinking in Hong Kong (drinking prevalence regardless of frequency: $55.4 \%$ in men and $19.4 \%$ in women) ${ }^{16}$ is not as common as in Western countries such as the UK (92.3\% in males and $87.8 \%$ in females aged $16-24$ years) ${ }^{2}$ drinking has become more socially acceptable due to heavy advertising portraying alcohol drinking, particularly wine drinking, as stylish and high class, and after beer and wine tax was abolished in $2008 .^{21}{ }^{22}$ The sociodemographic factors of adolescent drinking probably differ by drinking culture and norms in different populations. The present study investigated the association of sociodemographic characteristics with current drinking, binge drinking and the type of alcohol consumed in Hong Kong adolescents. The findings from Hong Kong may also have implications for countries where drinking prevalence is relatively low but increasing with economic development. ${ }^{23} 24$

\section{METHODS}

\section{Study design}

In 2012-2013, a school-based alcohol survey was conducted in Hong Kong. A total of 44 secondary schools were randomly selected from all the five regions of Hong Kong (Hong Kong Island 4, Kowloon East 8, Kowloon West 10, New Territory 
East 13 and New Territory West 12) in proportion to the number of schools in each region. These schools covered 16 of the 18 administrative districts of Hong Kong and were comparable with all schools in the source of funding (subsidised 79\% in sample vs $79 \%$ in Hong Kong, direct subsidy $16 \%$ vs $13 \%$, Government $2.3 \%$ vs $7 \%$, private $2.3 \%$ vs $1 \%$ ), among these, one international school was also included. All secondary 1 (US grade 7) to 6 students in the selected schools were invited. An invitation letter was given to parents, and declining parents were asked to have their child return a blank questionnaire during the survey. Even with parental consent, student participation was still voluntary. Students were asked to complete an anonymous questionnaire during a class period. Teachers helped put the questionnaires in an opaque envelope immediately after completion. All questionnaires were sent back to the University of Hong Kong by our research team. Teachers were instructed to avoid looking at the students' answers. The cover page of the questionnaire booklet and the anonymity of the survey also prevented the teachers from knowing individual responses. Therefore, it was unlikely that students would misreport. Ethical approval was obtained from the Institutional Review Board of the University of Hong Kong/Hospital Authority Hong Kong West Cluster.

\section{Subjects}

Of 23096 (91.0\% of 25381 invited) students returning the questionnaires, 21627 (94\%) remained for analysis (mean age 14.7 years, age range $11-20$ years, SD $1.8 ; 51.6 \%$ boys) after excluding those with information on sex or age missing, and excluding those with over half their questionnaire answers missing or with conflicting information regarding the frequency of alcohol drinking. Students remaining in analyses were similar to the original sample in sex $(51.6 \%$ and $52.1 \%$ boys, respectively) and age (mean age 14.7 years, SD 1.8 years for both samples). Cohen effect size between the two samples was 0.1 for sex and 0.2 for age, indicating small differences.

\section{Measurements}

Students were asked the question, 'In the past 30 days, on how many days did you drink alcohol?'. Respondents were categorised as current drinkers if they drank on at least one day regardless of the amount. Binge drinking was assessed by the question, 'In the past 30 days, on how many days have you drunk at least five drinks in a row?', with one drink defined as ' $330 \mathrm{~mL}$ of beer, $120 \mathrm{~mL}$ of wine or $30 \mathrm{~mL}$ of spirits'. Current drinking and binge drinking are both common classifications reporting at least 1 day of such drinking were defined as binge drinkers. Those who had never drunk alcohol were defined as never-drinkers. Students were asked to report the most common type of alcohol they drank, with response options of 'I do not drink', 'fruit wine', 'beer', 'wine', 'Chinese wine', 'spirits' and other types. A new variable was generated with five categories of 'never drinker', 'preference of fruit wine', 'preference of beer', 'preference of wine' and 'preference of spirits'. Since few students chose the option of 'Chinese wine' $(\mathrm{N}=162)$, Chinese wine was not included in the analysis. Alcohol Use Dependence Identification Test (AUDIT) was also included in the questionnaire, but the number of respondents with alcohol dependence $(1.1 \%)$ was too small for analysis as an outcome.

Sex, age, place of birth, perceived family affluence, type of housing and family structure were broadly included as sociodemographic factors. Age was categorised as ('14 years or below' and ' 15 years or above'); place of birth as born in Hong Kong, used in adolescent epidemiological studies. ${ }^{25}$ Respondents

Mainland China or other places; perceived family affluence as low, medium and high; type of housing as public rental housing, subsidised private housing, private housing and temporary/ others/unknown; and family structure was categorised as parents married, separated or divorced, or one or both parents deceased.

\section{Statistical analyses}

Descriptive analyses were used to describe sociodemographic and drinking behaviour variables in the total sample. Prevalence of current and binge drinking was weighted based on sex, age and grade distribution of the underlying adolescent population, using data provided by the Census and Statistics Department of Hong Kong. Pearson's $\chi^{2}$ tests were used to test univariate relations between sociodemographic characteristics and drinking behaviours. Multilevel analysis is an effective method to use when dealing with the hierarchical structure of data (student being level 1 and school being level 2) and to adjust for school clustering effects (students nested within the same school may have common characteristics). ${ }^{26}$ Multilevel logistic regression with random intercept was used to examine the association of various sociodemographic factors with current drinking and binge drinking in separate models employing the 'xtmelogit' function in STATA V.13.0. The OR and corresponding 95\% CI were generated. Additionally, a likelihood ratio test was performed to test the goodness of model fit between multilevel regression and traditional logistic regression. $p$ Value of $<0.05$ indicated superiority of multilevel regression to the traditional logistic regression.

\section{Table 1 Background characteristics}

\begin{tabular}{|c|c|c|}
\hline & $\mathbf{N}$ & Per cent \\
\hline \multicolumn{3}{|l|}{ Sex } \\
\hline Boys & 11168 & 51.6 \\
\hline Girls & 10459 & 48.4 \\
\hline \multicolumn{3}{|l|}{ Age, years } \\
\hline$\leq 14$ & 2866 & 13.4 \\
\hline$\geq 15$ & 3356 & 15.5 \\
\hline \multicolumn{3}{|l|}{ Place of birth } \\
\hline Hong Kong & 16220 & 75.3 \\
\hline Mainland China & 4497 & 20.9 \\
\hline Other places & 819 & 3.8 \\
\hline \multicolumn{3}{|l|}{ Perceived family affluence } \\
\hline Low & 6046 & 28.4 \\
\hline Medium & 12221 & 57.4 \\
\hline High & 3016 & 14.2 \\
\hline \multicolumn{3}{|l|}{ Type of housing } \\
\hline Public rental housing & 8397 & 39.3 \\
\hline Subsidised private housing & 2789 & 13.0 \\
\hline Private housing & 7480 & 35.0 \\
\hline Temporary/others/unknown & 2706 & 12.7 \\
\hline \multicolumn{3}{|l|}{ Family structure } \\
\hline Parents married & 17601 & 82.5 \\
\hline Parents separated or divorced & 2650 & 12.4 \\
\hline One or both parents deceased & 1082 & 5.1 \\
\hline \multicolumn{3}{|l|}{ Current drinking } \\
\hline No & 17015 & 78.8 \\
\hline Yes & 4570 & 21.2 \\
\hline \multicolumn{3}{|l|}{ Binge drinking } \\
\hline No & 20010 & 92.9 \\
\hline Yes & 1527 & 7.1 \\
\hline
\end{tabular}


As the 'xtmelogit' function is not applicable when the outcome variable is multinomial, we used traditional multinomial logit regression to examine the association between main alcohol type and sociodemographic characteristics with the option of 'robust clust (school variable)' to adjust for school clustering in STATA V.13.0. Multinomial logit regression was used to examine the association between sociodemographic characteristics and the type of alcohol consumed. Multilevel analysis is an effective method to use when dealing with the multilevel structure of data (student being level 1 and school being level 2) and to adjust for school clustering effects. ${ }^{26}$ Parental drinking could be an intermediate variable of the studied association, so it was not adjusted for.

\section{RESULTS}

Table 1 shows that most students were born in Hong Kong (75.3\%), perceived their family affluence as medium $(57.4 \%)$ and had an intact family (parents remained married) $(82.5 \%)$. Public rental housing (government subsidised) was most commonly reported (39.3\%), followed by private housing (35.0\%) and home ownership scheme (private with government subsidy) (13.0\%). One in $5(21.2 \%)$ adolescents were current drinkers and $7.1 \%$ were binge drinkers.

Table 2 shows that older age, higher perceived family affluence, private housing and non-intact family structure were associated with current alcohol drinking. After mutual adjustment and adjusting for school clustering effect, current alcohol drinking was significantly associated with older age (adjusted OR, AOR, $1.87,95 \%$ CI 1.74 to 2.02 ), medium family affluence $(1.11,1.02$ to 1.21$)$, high family affluence $(1.55,1.38$ to 1.75 , $\mathrm{p}$ for trend $<0.001)$, private housing $(1.11,1.02$ to 1.21$)$, separated/divorced parents $(1.31,1.19$ to 1.45$)$ and one or both parents deceased (1.40, 1.21 to 1.62$)$.

Table 3 shows the risk factors of binge drinking were the same as those of current drinking except for housing type, which became non-significant, and being born in Mainland China, which became significant. Being older (AOR per year $1.29,1.25$ to 1.33$)$, high family affluence $(1.53,1.28$ to 1.83$)$, separated/divorced parents $(1.47,1.27$ to 1.71$)$ and one or both deceased parents $(1.99,1.63$ to 2.43$)$ were positively associated with binge drinking, while being born in Mainland China was negatively associated with binge drinking $(0.81,0.70$ to 0.94$)$. These risk factors generally had stronger associations with binge drinking than with current drinking.

Table 4 shows that beer was the most popular type of alcohol (18.1\%), followed by fruit wine $(12.2 \%)$, wine $(7.2 \%)$ and spirits (1.6\%). Generally, compared with never-drinkers, older age and separated/divorced parents were significantly associated with each type of alcohol consumed. Girls were more likely to drink fruit wine (AOR 1.48, 95\% CI 1.36 to 1.62), but less likely to drink beer $(0.85,0.79$ to 0.92$)$ and spirits $(0.69,0.54$ to 0.87$)$. Being born in Mainland China was associated with drinking beer $(1.19,1.08$ to 1.31$)$. Students who reported high family affluence were more likely to drink wine and spirits. Similarly, private housing was associated with drinking wine $(1.36,1.18$ to 1.56$)$.

\section{DISCUSSION}

We found adolescent current drinking associated with older age, higher perceived family affluence, private housing and having one or both parents deceased. Being older as a risk factor of adolescent drinking has been commonly reported by other

Table 2 Sociodemographic factors associated with current alcohol drinking

\begin{tabular}{|c|c|c|c|}
\hline & Current drinking, $\mathrm{N}(\%)$ & Crude OR $(95 \% \mathrm{Cl})$ & Adjusted OR† (95\% Cl) \\
\hline \multicolumn{4}{|l|}{ Age, years } \\
\hline$\leq 14$ & $1559(15.8)$ & 1 & 1 \\
\hline$\geq 15$ & $3011(25.7)$ & $1.79(1.70 \text { to } 1.92)^{* * *}$ & $1.87(1.74 \text { to } 2.02)^{* * *}$ \\
\hline \multicolumn{4}{|l|}{ Sex } \\
\hline Boys & $2441(21.9)$ & 1 & 1 \\
\hline Girls & $2129(20.4)$ & $0.99(0.93 \text { to } 1.07)^{* *}$ & 0.99 (0.92 to 1.06$)$ \\
\hline \multicolumn{4}{|l|}{ Place of birth } \\
\hline Hong Kong & $3429(21.2)$ & 1 & 1 \\
\hline Mainland China & $934(20.8)$ & $0.94(0.87$ to 1.03$)$ & $0.92(0.84$ to 1.01$)$ \\
\hline Other places & $186(22.8)$ & $1.08(0.90$ to 1.29$)$ & 1.01 (0.84 to 1.22$)$ \\
\hline \multicolumn{4}{|l|}{ Perceived family affluence } \\
\hline Low & $1206(20.0)$ & 1 & 1 \\
\hline Medium & $2498(20.5)$ & $1.03(0.95$ to 1.11$)$ & $1.11(1.02 \text { to } 1.21)^{*}$ \\
\hline High & 785 (26.1) & $1.42(1.28 \text { to } 1.59)^{\star * *}$ & $1.55(1.38 \text { to } 1.75)^{* * *}$ \\
\hline$p$ for trend & & $<0.001$ & $<0.001$ \\
\hline \multicolumn{4}{|l|}{ Housing type } \\
\hline Public rental housing & $1672(19.9)$ & 1 & 1 \\
\hline Subsidised private housing & $590(21.2)$ & $1.13(1.02 \text { to } 1.26)^{*}$ & 1.09 (0.97 to 1.22$)$ \\
\hline Private housing & $1750(23.4)$ & $1.09(1.14 \text { to } 1.29)^{* * *}$ & $1.11(1.01 \text { to } 1.21)^{*}$ \\
\hline Temporary/others/unknown & $506(18.8)$ & $0.88(0.79 \text { to } 0.99)^{*}$ & 0.91 (0.81 to 1.03$)$ \\
\hline \multicolumn{4}{|l|}{ Parental marital status } \\
\hline Married & $3560(20.3)$ & 1 & 1 \\
\hline Separated/divorced & $665(25.2)$ & $1.26(1.15 \text { to } 1.39)^{* * *}$ & $1.31(1.19 \text { to } 1.45)^{* * *}$ \\
\hline One or both parents deceased & $281(26.1)$ & $1.38(1.16 \text { to } 1.55)^{* * *}$ & $1.40(1.21 \text { to } 1.62)^{* * *}$ \\
\hline $\mathrm{p}$ Value from likelihood ratio test vs logistic regression & & $<0.01$ & $<0.001$ \\
\hline
\end{tabular}


Table 3 Sociodemographic factors associated with binge drinking

\begin{tabular}{|c|c|c|c|}
\hline Sociodemographic characteristics & Binge drinking, $\mathrm{N}(\%)$ & Crude OR $(95 \% \mathrm{Cl})$ & Adjusted OR $(95 \% \mathrm{Cl})$ \\
\hline \multicolumn{4}{|l|}{ Age, years } \\
\hline$\leq 14$ & $434(4.4)$ & 1 & 1 \\
\hline$\geq 15$ & $1093(9.3)$ & $2.11(1.87 \text { to } 2.38)^{* * *}$ & $2.20(1.94 \text { to } 2.49)^{* * *}$ \\
\hline \multicolumn{4}{|l|}{ Sex } \\
\hline Boys & $911(8.2)$ & 1 & 1 \\
\hline Girls & $616(5.9)$ & $0.82(0.73 \text { to } 0.92)^{* * *}$ & $0.81(0.73 \text { to } 0.91)^{* * *}$ \\
\hline \multicolumn{4}{|l|}{ Place of birth } \\
\hline Hong Kong & $1112(6.9)$ & 1 & 1 \\
\hline Mainland China & $313(7.0)$ & 0.88 (0.77 to 1.01$)$ & $0.81(0.70 \text { to } 0.94)^{* *}$ \\
\hline Other places & $95(11.7)$ & $1.54(1.20 \text { to } 1.97)^{* *}$ & $1.36(1.05 \text { to } 1.75)^{\star}$ \\
\hline \multicolumn{4}{|l|}{ Perceived family affluence } \\
\hline Low & $447(7.4)$ & 1 & 1 \\
\hline Medium & $768(6.3)$ & $0.85(0.75 \text { to } 0.96)^{* *}$ & $0.96(0.84$ to 1.10$)$ \\
\hline High & $286(9.5)$ & $1.31(1.11 \text { to } 1.55)^{* *}$ & $1.53(1.28 \text { to } 1.83)^{* * *}$ \\
\hline$p$ for trend & & 0.054 & $<0.001$ \\
\hline \multicolumn{4}{|l|}{ Housing type } \\
\hline Public rental housing & $569(6.8)$ & 1 & 1 \\
\hline Subsidised private housing & $157(5.7)$ & 0.94 (0.78 to 1.14$)$ & $0.92(0.75$ to 1.11$)$ \\
\hline Private housing & $560(7.5)$ & $1.08(0.94$ to 1.23$)$ & $1.01(0.87$ to 1.17$)$ \\
\hline Temporary/others/unknown & $224(8.3)$ & $1.08(0.91$ to 1.28$)$ & 1.12 (0.94 to 1.34$)$ \\
\hline \multicolumn{4}{|l|}{ Family structure } \\
\hline Married parents & $1096(6.3)$ & 1 & 1 \\
\hline Separated/divorced parents & $269(10.2)$ & $1.45(1.26 \text { to } 1.68)^{* * *}$ & $1.47(1.27 \text { to } 1.71)^{* * *}$ \\
\hline One or both parents deceased & 1079 (13.4) & $2.03(1.68 \text { to } 2.46)^{* * *}$ & $1.99(1.63 \text { to } 2.43)^{* * *}$ \\
\hline p Value from likelihood ratio test vs logistic regression & & $<0.001$ & $<0.001$ \\
\hline
\end{tabular}

epidemiological studies. ${ }^{8}$ Older adolescents may be more stressed and believe that drinking could help them cope with problems. ${ }^{17} 27$ However, older adolescents may have more exposure to drinking behaviours of others, such as parents and peers, as well as to advertising of alcohol drinking in the media. They may also more frequently attend social gatherings where alcohol drinking is deemed necessary for fitting into a group. $^{17} 28$

We found no sex difference in current drinking but binge drinking was more common in boys. That heavy drinking is predominated by males has commonly been reported. ${ }^{29}$ Boys were also reportedly more likely than girls to take part in other risk behaviours, such as smoking and illicit drug use. ${ }^{30}$ However, binge drinking prevalence was similar in adolescent boys and girls in the US, with current drinking more common in girls. ${ }^{4}$ Lower alcohol drinking in girls was also found in Hong Kong college students. ${ }^{31}$ It has been reported that Hong Kong male adolescents commonly drank to feel the effect of alcohol while females drank to have fun. ${ }^{32}$ Such different drinking motives may partly explain the higher prevalence of binge drinking in the adolescent boys in this study.

Higher socioeconomic status (SES) in general, as proxied by higher perceived family affluence and private housing, was associated with current drinking in the present study. These findings were consistent with those in studies using parental professional occupation as an indicator of SES. ${ }^{12} 33$ However, these results were contrary to those from studies on adolescent smoking and drug use in which lower SES was consistently a risk factor. ${ }^{30} 34$ This may suggest that alcohol drinking is considered acceptable for adolescents, ${ }^{35}$ without the negative images typically associated with substance use. This is consistent with the positive image promoted directly by the alcohol industry and indirectly by the government through waiving beer and wine tax. We have also reported that Hong Kong parents with higher family affluence or parents who had higher education levels were more likely to mention the benefits of alcohol and that alcohol tastes good, and more willing to ask their children to open a bottle of alcohol and pour it. ${ }^{36}$ Higher SES was also associated with binge drinking in the present study. It is unlikely that parents, especially those with high SES, would accept or encourage adolescent binge drinking. The observed association may reflect that adolescent drinking is prone to develop into binge drinking, highlighting the risk of introducing alcohol to adolescents even in families with higher SES.

Students who were born in Mainland China were less likely to be binge drinkers compared with those born in Hong Kong. Previous research shows that many of the new arrivals from Mainland China to Hong Kong through cross-boundary marriage were women and most of them were housewives, ${ }^{37}$ and families of cross-boundary marriage may have lower family affluence. Therefore, this association is consistent with that of higher SES with more alcohol drinking.

Adolescents from non-intact families were more likely to be current drinkers in the present study. Non-intact family structure has been associated with smoking due to insufficient support and control, less family attachment, and less monitoring and discipline. $^{13} 3839$ Our results were consistent with those of other studies reporting that non-intact family structure was associated with alcohol drinking among adolescents due to low family attachment or insufficient parent-child communication. ${ }^{13}{ }^{14}$ However, different results have been reported of living with both parents not being associated with reduced regular drinking in adolescents aged 14-15 years. ${ }^{15}$ This discrepancy may have risen due to different alcohol-specific rules by parents. Children 
Table 4 Association between sociodemographic factors and main type of alcohol consumed (fruit wine, beer, wine and spirits)

\begin{tabular}{|c|c|c|c|c|}
\hline & \multicolumn{4}{|c|}{ Adjusted OR (95\% CI) (reference category: never-drinkers) } \\
\hline & Fruit wine & Beer & Wine & Spirits \\
\hline Prevalence, N (\%) & $2503(12.2)$ & $3699(18.1)$ & $1464(7.2)$ & $326(1.6)$ \\
\hline \multicolumn{5}{|l|}{ Sex } \\
\hline Boys & 1 & 1 & 1 & 1 \\
\hline Girls & $1.48(1.36 \text { to } 1.62)^{* * *}$ & $0.85(0.79 \text { to } 0.92)^{* * *}$ & 0.99 (0.89 to 1.11$)$ & $0.69(0.54 \text { to } 0.87)^{* *}$ \\
\hline \multicolumn{5}{|l|}{ Age, years } \\
\hline$\leq 14$ & 1 & 1 & 1 & 1 \\
\hline$\geq 15$ & $1.19(1.16 \text { to } 1.22)^{* * *}$ & $1.36(1.33 \text { to } 1.39)^{* * *}$ & $1.28(1.24 \text { to } 1.33)^{* *}$ & $1.62(1.52 \text { to } 1.74)^{* * *}$ \\
\hline \multicolumn{5}{|l|}{ Place of birth } \\
\hline Hong Kong & 1 & 1 & 1 & 1 \\
\hline Mainland China & $1.01(0.90$ to 1.13$)$ & $1.19(1.08 \text { to } 1.31)^{*}$ & $1.09(0.95$ to 1.26$)$ & 1.01 (0.76 to 1.34$)$ \\
\hline Other places & $0.56(0.46 \text { to } 0.70)^{* * *}$ & $0.69(0.54 \text { to } 0.87)^{* *}$ & $0.69(0.50 \text { to } 0.95)^{*}$ & $1.95(1.27 \text { to } 2.99)^{* *}$ \\
\hline \multicolumn{5}{|l|}{ Perceived family affluence } \\
\hline Low & 1 & 1 & 1 & 1 \\
\hline Medium & 0.96 (0.87 to 1.07$)$ & 1.07 (0.98 to 1.17$)$ & $1.06(0.93$ to 1.22$)$ & $1.28(0.97$ to 1.70$)$ \\
\hline High & $1.03(0.88$ to 1.21$)$ & 1.11 (0.97 to 1.28$)$ & $1.91(1.59 \text { to } 2.30)^{* * *}$ & $2.23(1.54 \text { to } 3.24)^{* * *}$ \\
\hline $\mathrm{p}$ for trend & NA & $<0.001$ & $<0.001$ & $<0.001$ \\
\hline \multicolumn{5}{|l|}{ Housing type } \\
\hline Public rental housing & 1 & 1 & 1 & 1 \\
\hline Subsidised private housing & $1.19(1.04 \text { to } 1.37)^{*}$ & 1.00 (0.89 to 1.14$)$ & $1.11(0.92$ to 1.33$)$ & 0.99 (0.67 to 1.48$)$ \\
\hline Private housing & $1.02(0.92$ to 1.14$)$ & 1.07 (0.97 to 1.17$)$ & $1.36(1.18 \text { to } 1.56)^{* * *}$ & 1.21 (0.91 to 1.62 ) \\
\hline Temporary/others/unknown & $0.75(0.64 \text { to } 0.88)^{* * *}$ & $0.86(0.75 \text { to } 0.98)^{*}$ & 1.01 (0.83 to 1.23$)$ & 1.39 (0.97 to 1.98$)$ \\
\hline \multicolumn{5}{|l|}{ Family structure } \\
\hline Married parents & 1 & 1 & 1 & 1 \\
\hline Separated/divorced parents & $1.23(1.07 \text { to } 1.40)^{* *}$ & $1.55(1.38 \text { to } 1.73)^{* * *}$ & $1.31(1.10 \text { to } 1.55)^{* *}$ & $1.97(1.44 \text { to } 2.69)^{* * *}$ \\
\hline One or both parents deceased & 1.05 (0.83 to 1.34$)$ & $1.16(0.95$ to 1.42$)$ & $1.40(1.06 \text { to } 1.85)^{*}$ & $3.35(2.22 \text { to } 5.05)^{* * *}$ \\
\hline
\end{tabular}

${ }^{*} p<0.05,{ }^{* *} p<0.01$ and ${ }^{* * *} p<0.001$.

may be less likely to drink under strict alcohol-specific rules despite non-intact families. ${ }^{40}$ Although we lack information on whether students had a stepfamily in our study, it has been shown that adolescents from stepfamilies had even higher likelihood of smoking than those from lone-parent families and intact families. ${ }^{38}$

As a cross-sectional study, the major weakness of the present study is that temporal sequence of associations cannot be ascertained. Using perceived family affluence and housing type to indicate SES might not be complete without including family income, but family income may be sensitive and difficult for adolescents to report reliably. ${ }^{41}$

To the best of our knowledge, this is the first study on the sociodemographic risk factors of adolescent drinking by alcohol type. Girls were more likely to choose fruit wine, but boys were more likely to choose beer and spirits. Fruit wine may attract girls by sporting colourful label designs and by its relatively low alcohol concentration. However, girls should be discouraged to drink any type of alcohol. Higher perceived family affluence was associated with all types of alcohol studied, but significant results were obtained only for wine and spirits. This could partly be explained by the high-class image of wine and spirit advertised by the alcohol industry. Adolescents who prefer higher alcohol concentration beverages may be more risk-taking, which puts them at particularly high risk for alcohol dependence later in life. Our findings may help identify beer and spirits drinkers and may lead to the design of better tailored alcohol interventions. Further studies are warranted to ascertain if preferring higher concentration alcohol beverages is associated with alcohol dependence in later life.

\section{CONCLUSIONS}

Generally, adolescents who were older and had higher SES were more likely to be current and binge drinkers. High family affluence was associated with wine and spirits drinking. Beer and spirits were more preferred by boys than by girls, and fruit wine was more favoured by girls than by boys. These results indicated high-risk groups for adolescent alcohol interventions.

\section{What is already known on this subject}

Adolescent drinking in Western countries has been associated with older age and high socioeconomic status, but the association with family structure was inconsistent. Little is known about the association between sociodemographic characteristics and preference of alcohol types, such as beer, wine and spirits.

\section{What this study adds}

In Hong Kong adolescents, older age, non-intact family structure and higher socioeconomic status was associated with binge drinking and current drinking, especially with wine and spirits drinking. Beer and spirits were preferred more by boys, and fruit wine by girls. 
Acknowledgements The authors would like to thank the Health and Medical Research Fund (10111241) for financial support, and the schools and students for their participation.

Contributors RH, MPW, WSL and SYH conceptualised and designed the study. $\mathrm{RH}$ conducted the statistical analysis and drafted the manuscript. SYH made significant contribution to the data analysis. All the authors contributed to the manuscript revision and approved the final manuscript.

Competing interests None declared.

Patient consent Obtained.

Ethics approval Institutional Review Board of the University of Hong Kong/ Hospital Authority Hong Kong West Cluster.

Provenance and peer review Not commissioned; externally peer reviewed.

\section{REFERENCES}

1 Powell PA, Faden VB, Wing S. The surgeon general's call to action to prevent and reduce underage drinking, 2007. US Department of Health and Human Services, 2007.

2 Gill JS. Reported levels of alcohol consumption and binge drinking within the UK undergraduate student population over the last 25 years. AlcoholAlcohol 2002;37:109-20.

3 Arata CM, Stafford J, Tims MS. High school drinking and its consequences. Adolescence 2002;38:567-79.

4 Miller JW, Naimi TS, Brewer RD, et al. Binge drinking and associated health risk behaviors among high school students. Pediatrics 2007;119:76-85.

5 Guerri C, Pascual M. Mechanisms involved in the neurotoxic, cognitive, and neurobehavioral effects of alcohol consumption during adolescence. Alcohol 2010;44:15-26.

6 Wang J, Patten SB. Prospective study of frequent heavy alcohol use and the risk of major depression in the Canadian general population. Depress Anxiety 2002;15:42-5.

7 American Academy of Pediatrics. Alcohol use and abuse: a pediatric concern. Pediatrics 2001;108:185-9.

8 Kokotailo P. Alcohol use by youth and adolescents: a pediatric concern. Pediatrics 2010;125:1078-87.

9 Grant BF, Dawson DA. Age at onset of alcohol use and its association with DSM-IV alcohol abuse and dependence: results from the National Longitudinal Alcohol Epidemiologic Survey. J Subst Abuse 1997;9:103-10.

10 Gallimberti L, Chindamo S, Buja A, et al. Underage drinking on Saturday nights, sociodemographic and environmental risk factors: a cross-sectional study. Subst Abuse Treat Prev Policy 2011;6:15.

11 Tomcikova Z, Madarasova Geckova A, Van Dijk JP, et al. Characteristics of adolescent excessive drinkers compared with consumers and abstainers. Drug Alcohol Rev 2011:30:157-65.

12 Zucker RA, Harford TC. National study of the demography of adolescent drinking practices in 1980. J Stud Alcohol 1983;44:974.

13 Sokol-Katz J, Dunham R, Zimmerman R. Family structure versus parental attachment in controlling adolescent deviant behavior: a social control model. Adolescence 1997;32:199-215.

14 Mak KK, Ho SY, Thomas GN, et al. Family structure, parent-child conversation time and substance use among Chinese adolescents. BMC Public Health 2010;10:503

15 McArdle P, Wiegersma A, Gilvarry E, et al. European adolescent substance use: the roles of family structure, function and gender. Addiction 2002;97: 329-36.

16 Janghorbani M, Ho SY, Lam TH, et al. Prevalence and correlates of alcohol use: a population-based study in Hong Kong. Addiction 2003;98:215-24.

17 Kuntsche E, Knibbe R, Gmel G, et al. 'I drink spirits to get drunk and block out my problems...'beverage preference, drinking motives and alcohol use in adolescence. Alcohol Alcohol 2006;41:566-73.
18 Fox RJ, Krugman DM, Fletcher JE, et al. Adolescents' attention to beer and cigarette print ads and associated product warnings. J Advertising 1998;27:57-68.

19 White V, Bariola E. "E. Australian secondary school students' use of tobacco, alcohol, and over-the counter and illicit substances in 2011. Report prepared for: Drug Strategy Branch Australian Government Department of Health and Ageing. December 2012." 2013:118.

20 Smart RG. Behavioral and social consequences related to the consumption of different beverage types. J Stud Alcohol 1996;57:77-84.

21 Leung SF, Arthur D. The alcohol use disorders identification test (AUDIT): validation of an instrument for enhancing nursing practice in Hong Kong. Int J Nurs Stud 2000; $37: 57-64$

22 The Government of the Hong Kong Special Administrative Region. 2008-9 Budget highlight. http://www.budget.gov.hk/2008/eng/highlights.html on 9 Sep 2015.

23 Zhang J, Casswell S, Cai H. Increased drinking in a metropolitan city in China: a study of alcohol consumption patterns and changes. Addiction 2008;103:416-23.

24 Yang G, Wang Y, Zeng Y, et al. Rapid health transition in China, 1990-2010: findings from the Global Burden of Disease Study 2010. Lancet 2013;381:1987-2015.

25 Windle M. Alcohol use among adolescents and young adults. Population 2003:45.5.9:19-5.

26 Twisk JW. Applied multilevel analysis: a practical guide for medical researchers. Cambridge: University Press, 2006.

27 Kuntsche $E$, Knibbe R, Gmel G, et al. Why do young people drink? A review of drinking motives. Clin Psychol Rev 2005;25:841-61.

28 Blumenthal H, Leen-Feldner EW, Frala JL, et al. Social anxiety and motives for alcohol use among adolescents. Psychol Addict Behav 2010;24:529.

29 Wilsnack RW, Vogeltanz ND, Wilsnack SC, et al. Gender differences in alcohol consumption and adverse drinking consequences: cross-cultural patterns. Addiction 2000;95:251-65

30 Lai MK, Ho SY, Lam TH. Perceived peer smoking prevalence and its association with smoking behaviours and intentions in Hong Kong Chinese adolescents. Addiction 2004;99:1195-205.

31 Tse EC. A comparative analysis of alcohol consumption pattern among global university students. Paper presented at the 2011 Annual Conference of the International Council on Hotel, Restaurant, and Institutional Education. Denver, Colorado, 27-30 July 2011.

32 Lo CC, Globetti G. Gender differences in drinking patterns among Hong Kong Chinese youth: a pilot study. Subst Use Misuse 2000;35:1297-306.

33 Murray DM, Richards PS, Luepker RV, et al. The prevention of cigarette smoking in children: two-and three-year follow-up comparisons of four prevention strategies. J Behav Med 1987;10:595-611.

34 Pampel FC, Krueger PM, Denney JT. Socioeconomic disparities in health behaviors Ann Rev Soc 2010;36:349.

35 Ettner SL. New evidence on the relationship between income and health. J Health Econ 1996:15:67-85.

36 Au WM, Ho SY, Wang MP, et al. Correlates of pro-drinking practices among parents of adolescents. PLOS ONE 2015;10:e0119554.

37 Central Policy Unit of The Government of The Hong Kong Special Administrative Region. Cross-boundary marriage in Hong Kong: causes and consequences. 2013. http://www.cpu.gov.hk/doc/en/research_reports/executive_summary_cross_ boundary_marriages_in_hong_kong_en.pdf (accessed 28 Jul 2015).

38 Griesbach D, Amos A, Currie C. Adolescent smoking and family structure in Europe. Soc Sci Med 2003;56:41-52.

39 Bianchi SM. Understanding differences between divorced and intact families. J Marriage and Family 1997;59:779.

40 van den Eijnden $\mathrm{R}$, Mheen DV, Vet $\mathrm{R}$, et al. Alcohol-specific parenting and adolescents' alcohol-related problems: the interacting role of alcohol availability at home and parental rules. J Stud Alcohol Drugs 2011;72:408.

41 Currie CE, Elton RA, Todd J, et al. Indicators of socioeconomic status for adolescents: the WHO Health Behaviour in School-aged Children Survey. Health Educ Res 1997;12:385-97. 